\title{
Strengthening intergenerational connections
}

\author{
The pandemic has highlighted the need for stronger intergenerational connections. Restarting intergenerational \\ programs and expanding newer initiatives to connect people of different ages must be prioritized in debates about \\ how society should progress post-pandemic.
}

$\mathrm{S}$ trong intergenerational relationships were the norm for most of human evolution, both within and between families, and essential for human survival and socialization. The last 50 years,

however, have seen a growing trend towards age segregation in various facets of life, including school education, the workplace and within the extended family and broader social life. Increased age segregation over the human life course has led to more entrenched ageist stereotyping and reduced opportunities for different generations to learn from one another, and may contribute to social isolation, poorer health and a lower quality of life for some older people.

Beginning in the 1960s, many types of intergenerational programs were launched to mitigate the negative consequences of age segregation. These programs improve 'generational intelligence', or the ability to appreciate how people from other generations experience the world, and incorporate this understanding into behavior and decision-making. Many initiatives to promote age integration have been successful in tackling a range of broad social issues. Japan introduced intergenerational care schemes in the 1970s, and similar schemes have been adopted by other countries. Intergenerational care schemes can bring together older people with school children through mentoring programs (https://bit.ly/3diVZm2) or more integrated spaces (https://bit.ly/3sxyFr0) that physically combine nurseries with care homes. Intergenerational mixing via these programs improves literacy and social skills in children while reducing reports of loneliness in the older participants. In England, the 'Grandmentors' (https://bit. ly/39JZH7p) initiative by Volunteering Matters connects young people leaving foster or residential care with older volunteers, who help them navigate the challenges of living independently. Given the obvious mutual benefits of these intergenerational programs, the think tank United for All Ages is campaigning (https:// bit.ly/3m3xiOt) for 1,000 cross-generational sites to be opened by 2030 in the UK.

In addition to educational schemes that encourage interaction between people at either end of the lifespan, many businesses are looking to foster improved communication and productive collaboration between younger and older workers. A key motivation for this is the opportunity to preserve institutional memory (https://bbc. in/3fr7GJX) and facilitate workers passing on what they learned during their employment; in particular, the reasoning behind the design of longer-term projects and work systems, and how common obstacles were overcome in their development. The bidirectional benefits of multigenerational workforces (https:// bit.ly/31wWVxD) are being recognized and may require active encouragement of age integration strategies such as reverse mentoring (https://bit.ly/3u0YVKG), where younger workers share their skills and perspectives with older colleagues. CIRKEL (https://cirkel.co/about) is a particularly interesting initiative that organizes mixing events and introduces people from different generations according to their specific mentoring or networking needs for personal and professional development.

The pandemic has paused many of these intergenerational mixing schemes at a time when this form of interaction and the empathy it can nurture are even more critical, as the pandemic impacts different age groups in different ways. For example, younger people are more affected by the economic and mental health consequences (https:// bit.ly/3dk8g9N) of lockdown restrictions, whereas older people were presumed to be more at risk of extreme social isolation in addition to facing greater health risks from the virus itself. On occasion, intergenerational conflict appears to have been stoked by the media (https://bit.ly/3u4Bqk0) and some politicians. Different age groups have been blamed for not following public health guidelines, and there are debates over how to best protect physically vulnerable groups while mitigating social and economic consequences (https://bit.ly/3rBuvNt) for younger people. However, the lockdown restrictions have been accompanied by promising signs of other innovative types of intergenerational mixing. Research (https:// prn.to/2O5DqsS) has shown an increase in communication between younger and older people, with $60 \%$ of people reporting that they have been speaking on the telephone more with relatives and others of different generations during the pandemic for mutual emotional support and skill sharing. Over three-quarters of people in the UK in one survey indicated that they hope to spend more time with people of other generations once the pandemic has abated. Likewise, mutual aid groups (Qual. Ageing 21, 253-259; 2020) have sprung up during the pandemic that foster interaction and solidarity between people of different generations. In recognition of the need to publicize the benefits of age integration, the first 'National Intergenerational Week' was planned by the St. Monica's Trust in the UK for March 2020, but was also stymied by the sudden lockdown. The online campaign in March 2021 was organized by Generations Working Together (https://bit.ly/3m4as9n) to highlight ongoing projects and publicize new ideas that foster communication and shared experiences between people from different age groups. Many of these schemes were adapted to operate remotely and also emphasize that intergenerational mixing can be about simply having fun together (https://bit.ly/39sxBgN) as well as work and education.

Generations Working Together recently compiled a manifesto (https://bit. ly/39s60w8) for transforming Scotland into the first intergenerational country by 2030 , with guidelines for how to generate intergenerational spaces and a call for local government to consider intergenerational approaches more broadly during policy-making. The success of organizations such as Generations Working Together in building more intergenerational communities will depend both on sustaining public support and interest as well as proposing new feasible, innovative schemes for age integration and evidence of their immediate and longer-term community-wide benefits to policy-makers. The pandemic is a potent reminder of how we are all connected, and has opened up many debates about how we can reshape society for everyone's benefit in the future. How to restart and strengthen intergenerational programs should be a key part of these discussions.

Published online: 15 April 2021

https://doi.org/10.1038/s43587-021-00061-3 